IOS Press

\title{
Plasticity of body representations after surgical arm elongation in an achondroplasic patient
}

\author{
Rocco L. Cimmino ${ }^{\mathrm{a}, \mathrm{b}, *}$, Grazia Spitoni ${ }^{\mathrm{a}, \mathrm{b}}$, Andrea Serino ${ }^{\mathrm{c}}$, Gabriella Antonucci $^{\mathrm{a}, \mathrm{b}}$, Maurizio Catagni ${ }^{\mathrm{d}}$, \\ Marco Camagni ${ }^{\mathrm{d}}$, Patrick Haggard ${ }^{\mathrm{e}}$ and Luigi Pizzamiglio ${ }^{\mathrm{a}, \mathrm{b}}$ \\ ${ }^{a}$ Santa Lucia Foundation IRCCS, Rome, Italy \\ ${ }^{\mathrm{b}}$ Department of Psychology, University of Rome "La Sapienza", Rome, Italy \\ ${ }^{\mathrm{c}}$ Department of Psychology, Università degli Studi di Bologna, Bologna, Italy \\ ${ }^{\mathrm{d}}$ Department of Orthopaedic and Ilizarov Unit, Lecco General Hospital, Lecco, Italy \\ ${ }^{\mathrm{e}}$ Institute of Cognitive Neuroscience, University College London, London, UK
}

\begin{abstract}
.
Purpose: A realistic body representation needs to be constantly updated. In case of physiological modifications, body representations integrate information coming from different sensory sources, including the sense of touch. Previous studies described transient modifications of these representations following illusory distortions. In this single case study, we documented the changes occurred in lower-level, primary somatosensory, and higher-level representations, in a case of upper arms elongation. Method: We explored effects of arm lengthening on primary tactile perception (sensitivity and acuity), an implicit perceptual measure of body size (tactile distance judgement), body image (Daurat-Hmelijak test), and peri-personal space representation (audio-tactile interaction task).

Results: We show that patient's arm representation was changed after surgery. Specifically, we observed significant changes on tactile distance judgments, body image test and audio-tactile interaction task; also even though no changes were found on primary tactile perception a significant modification emerged in tactile acuity.

Conclusions: These findings are in line with evidence of cortical reorganization after arm elongation. They also support the view that the body representation of achondroplasics are modified after body-size reconstruction, and became similar to that of healthy controls.
\end{abstract}

Keywords: Body representations, peripersonal space, cortical plasticity, tactile perception, primary sensory processing

\section{Introduction}

Several lines of research with healthy volunteers and neuropsychological patients suggest that the brain maintains a range of representations of the body. While

*Corresponding author: Dr. Rocco Luca Cimmino, Santa Lucia Foundation IRCCS, Via Ardeatina 306, 00100 Roma, Italy. Tel.: +39 3294579290; E-mail: roccoluca.cimmino@uniroma1.it. several different models and classifications have been proposed (Schwoebel and Coslett, 2005), all agree on a broadly hierarchical arrangement. Lower-level representations are concerned essentially with the location of somatic stimuli (e.g., touch) on the body, while higher-level representations are concerned with the more global configuration of the body as a physical object, spatial relations between body parts, and identity and naming of body parts. Identifying these 
different body representations in experiments with healthy volunteers is difficult, since any specific bodyrelated stimulus used in testing will be processed by many or all of these representations. In contrast, neuropsychological studies of lesion patients have made important contributions to fractionating these different representations. For example, individual patients may show isolated deficits in peripersonal space representation (Làdavas and Serino, 2008), spatial configuration of body parts (Gerstmann, 1940, 1957), or in semantics of body parts (Semenza, 1988; Sirigu et al., 1991). However, inferences from patients are subject to both general and specific difficulties. First, there is a general difficulty in inferring normal brain organisation from cases of brain damage, because of non-focal damage, and possible compensatory adjustments (Basso et al., 1989; Levine and Mohr, 1979). Second, many lesions of parietal areas thought to house higher-order body representations also affect the postcentral areas that house low-level somatosensory function. Therefore, damage to high-level functions with intact low-level body representation is rare. Nevertheless, the causal nature of inference in lesion cases means that neuropsychological studies of body representation have had enduring influence. On the other hand, studies on healthy subjects have demonstrated that cortical body representations are not fixed entities, but are dynamic and continuously modified by experience. Most of these studies used tactile stimulation to measure body representations, and multisensory stimulation to illusorily manipulate them. For instance, in the so-called Pinocchio illusion (Lackner, 1988), a tendon vibration in the arm produces proprioceptive misinformation about its position and subsequent perceptual distortions regarding the size of the nose. Distorting the visual experience of one's own arm alters the perception of tactile distances on the arm (Taylor-Clarke et al., 2004); similarly, tactile distance perception on the finger is modified by vibration of the biceps and triceps, which induces an illusion of lengthening or shortening the index finger (de Vignemont et al., 2005). These findings suggest that the brain computes several sources of information (i.e. tactile, visual, proprioceptive) to scale information about skin contact in relationship to the perceived size of the body part tactilely stimulated. However, most previous experimental studies of body representation have been based on very brief illusory distortions in healthy volunteers, often lasting for a few minutes or less (de Vignemont et al., 2005).

\subsection{The case of physical body elongation}

In the present study, we aimed at overcoming the limitations of neuropsychological studies on brain damaged patients and of psychophysical studies on healthy volunteers by studying a new model of plasticity in an achondroplastic patient, MM, who underwent to a gradual, long lasting modification of her body, i.e. surgery with the Ilizarov technique (Ilizarov and Deviatov, 1971; Cattaneo et al., 1988) to increase the length of her upper limbs (see Fig. 1).

This procedure induces surgical lengthening of the arm, but leaves the afferent and efferent connections between the arm and the somatosensory cortices intact and the patients had no brain lesions. The technique also provides an ideal model for studying brain plasticity following a real, not illusory manipulation of the physical body structure. We investigated how surgery affected the representation of the elongated body part at several levels of tactile stimulus processing, that is, from primary somatosensation to higher levels of body representation. Based on previous findings ( $\mathrm{Di}$ Russo et al., 2006), we subjected MM to a battery of somatosensory and body representation tests immediately before the surgical elongation procedure (pre), at the end of the procedure (post, i.e. five months after the first evaluation, following removal of the elongation cage), and in a follow-up session performed six months later (follow-up). Low-level tactile perception was investigated using classical tests of detection and acuity (Von Frey's test and the two point discrimination task (2PDT)). Then to investigate how the brain computes intrinsic object properties from preliminary contact information, we used a distance discrimination task (DDt) for pairs of stimuli administered on the arm and on the neck as a reference site. This provided information about the metric representation of body parts. A pressure discrimination task (PDt) was used as a non-spatial control task. Besides processing sensory input from skin stimulation, the DDt also involves spatial computation of the position of incident points on the skin surface and the absolute length of the stimulated body part. We also investigated whether surgical elongation also affected the perceived configuration of the body as assessed by a more explicit visual task, specifically, the DauratHmeljiak task (DH) (Daurat-Hmeljiak et al., 1978). During the DH task, the patient was presented with individual tiles, each depicting a single body part (nine tiles: right/left leg-arm-hand-hemithorax and 


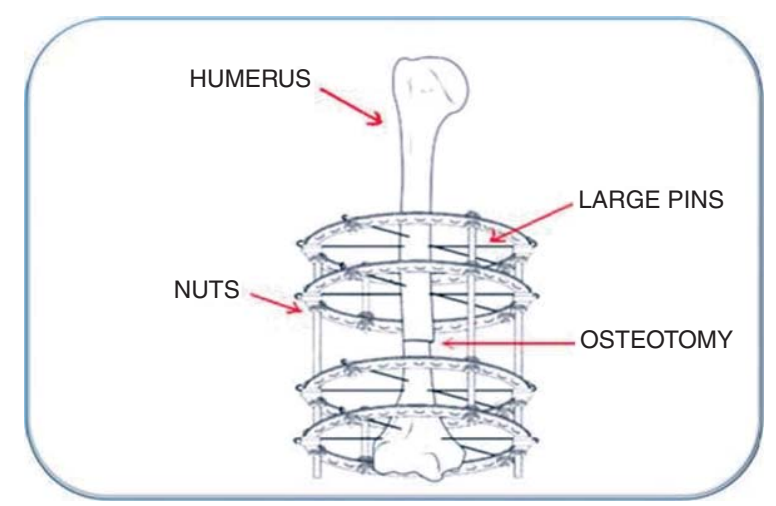

Fig. 1. Schematic representation of the Ilizarov bone-lengthening device. The cortex of the bone is partially cut, leaving the medulla intact; an external steel cage, fixed on the bones (fixation), progressively separates the two bone segments by about $1 \mathrm{~mm}$ a day. This progressive elongation prevents the formation of a callus and the physiological reconnection of the two parts of the bone. When the desired length is reached, the callus is allowed to solidify; the steel cage is removed only when the cortical structure of the two parts of the bone is consolidated.

the neck), and was asked to put each tile in the appropriate position on an empty board on which only the contour of a face was drawn to reproduce the shape of a standard body. The rationale of the task is that subjects refer to their own body representation to reproduce the shape of a human-like body.

\subsection{Changes in the peripersonal space}

Finally, we tested whether changes in the representation of the body also influence the representation of peripersonal space (PPS). PPS is the limited portion of space where we can physically interact with external objects, reachable by our upper limb. In order to represent PPS, our brain integrates information related to the position and size of the different body parts with information related to the location, size and movement of objects in space. Proprioceptive and tactile information related to the body are integrated with visual or acoustic information of the external objects present within the boundaries of peripersonal space (Rizzolatti et al., 1997, Graziano and Cooke, 2006, Làdavas and Serino, 2008). As these boundaries are usually defined by the physical dimensions of the body (Longo and Lourenco, 2007), PPS representation is likely affected by a change in the size of the physical body, and provides a valuable, implicit, and action-oriented test of body representation. We therefore investigated whether surgery for upper limb elongation affects the boundaries of PPS.
For this purpose, before and after surgery MM performed an audio-tactile interaction task that probed the extent of PPS around the upper limb.

\subsection{The timing of perceptual changes after surgery}

Overall, her performance on the sets of experiments showed that soon after surgery MM's arm perception was impaired with respect to that of age-matched healthy controls. Nevertheless, her performance significantly improved six months after the operation, approaching that of controls. This evidence supports the findings of a previous study on cortical reorganization after arm elongation (Di Russo et al., 2006); it also supports the view that achondroplasics' pre-existing body representation can be modified and become similar to that of healthy controls after modification of body size.

\section{Materials and method}

We studied how progressive limb elongation affects tactile perception, body and PPS representation in an achondroplasic woman who underwent surgical arm lengthening. At the time of the surgery, patient MM was 29 years old and her arm was elongated of about $10 \mathrm{~cm}$. At the age of 18 , thus 11 years before the present testing, she underwent surgical leg elongation $(13 \mathrm{~cm})$ and reached a height of $150 \mathrm{~cm}$. In the present study, MM was tested three times: before surgery (pre test), soon after the cages were removed (post test) and about one year after the surgery (follow-up). The control group included 26 age-matched participants (mean age $28, \mathrm{SD} \pm 1.15 ; 15$ female), all of them received tactile acuity assessment and DDt. Among them 20 subjects (mean age 29, $\mathrm{SD} \pm 1.08$ ) also received $\mathrm{PDt}$ task and DH. Due to technical problems four DH protocols were excluded from the analysis. Finally the control group for the PPS task consisted in seven participants (mean age 28, DS $\pm 1.83,5$ female). None of the participants had neurological or psychiatric diseases. The control group was tested twice, with a five-month interval between the first and the second evaluation. MM and healthy controls resulted strongly right-handed as measured by Edinburgh handedness inventory (EHI) (Oldfield, 1971). Each participant underwent five experimental assessments. In line with the ethical standards laid down in the 1964 Declaration 
of Helsinki, this study was approved the ethical committee of the IRCCS Fondazione Santa Lucia of Rome. All participants provided written informed consent prior to their inclusion in the study. Patient MM was recruited from the Manzoni Hospital in Lecco (Italy).

\subsection{Primary tactile tasks}

Tactile acuity was measured using Von Frey's test and the two point discrimination task (2PD). Von Frey's test is a classic measure of sensitivity to tactile pressure used for diagnosis or research (North Coast Medical, Inc., Morgan Hill, CA, USA). In this test, the tip of a fibre of a given weight (from 0.008 to 300 grams) is pressed against the skin at right angles. The force of application increases as long as the researcher continues to advance the probe, until the fibre bends. In this study, the procedure was repeated using differentweight fibres in both an ascending and a descending staircase. At each level of the staircase, 10 actual stimulation and 5 catch trials (a total of 15 stimulation) were presented. In each trial, the experimenter asked the participants whether they felt the stimulus, and they had to respond verbally. The threshold was established at the staircase level when the subjects reported 6 out of 10 stimuli correctly. Two-point discrimination thresholds were estimated by using an adjustable aesthesiometer (Med Core, St. Louis, MO, USA) with two spatially separated tips. Stimuli were manually delivered to the ventral skin of the arm just above the elbow, with the two stimuli points oriented vertically. Participants were blindfolded and were requested to discriminate between single and double taps and to respond verbally. In this procedure, double or single taps were given randomly. Only double taps were used to calculate the threshold. The separation between the two starting points were 1 and $5 \mathrm{~cm}$ in the ascending and descending mode, respectively. The separation was then reduced progressively by $1 \mathrm{~cm}$ after each correct response. When an error was made, the separation was subsequently increased by $1 \mathrm{~cm}$. The participants' threshold was derived from the minimum distance perceived between the two points five times consecutively.

\subsection{Distance discrimination task}

Stimuli consisted of two simultaneous contacts from a line of four miniature solenoids connected to a device (MSTC3-M\&E Solve, UK). The stimuli were fixed with malleable material and placed on the right humerus and neck. Stimuli administration was controlled by an electronic interface (NI-DAQ, 6800) connected to the computer and driven by a custommade code written in Lab-View (7.0). Two blocks of 108 trials were administered; in half of the trials, tactile distances were the same on the arm and the neck; in the other half, stimuli were more widely delivered on either the arm or the neck, with equal probability. To make the distance perceivable, we set the stimulators so that adjacent solenoids were separated $5 \mathrm{~mm}$ more than the subjective 2PD threshold; the same distance was used to place the solenoid on the neck (see Fig. 2). With this arrangement, three different distances could be compared between the stimulated body parts. Stimuli were always delivered first on the right arm and then (after $250 \mathrm{msec}$ ) on the neck. Subjects were then asked to verbally judge which of the two tactile distances they perceived as greater. The experimenter manually entered the response in the computer. Subjects received no feedback during the task.

\subsection{Pressure discrimination task}

The stimuli and protocol were the same as in the DDt, but here we focused on the pressure of each stimulation. Subjects perceived stimulations of different or equal intensity on the arm and on the neck and they had to say which stimulation was more intense. In each trial, stimuli intensity was set using the same softwarehardware device as in the DDt. Two blocks of 108 trials were administered, so that 3 different levels of intensity of the stimuli on the arm and the neck. Also in this case, in half of the trials stimuli intensity was the same for the two body parts, and in the other half stimuli were more intense on the arm or the neck, with equal probability.

\subsection{Body image test}

Subjects were asked to put one tile depicting a body part in the appropriate position on an empty board, where the contour of the model's face was drawn, in order to ideally reconstruct the model's entire body. As the tiles were all rectangular, there was nothing to suggest their correct location. After each trial, the previously placed tile was removed. Thus, to perform the task the participants had to refer to an internal representation of a human body image that depend on the representation of their own physical body. They performed the task seated at a desk in front of the 


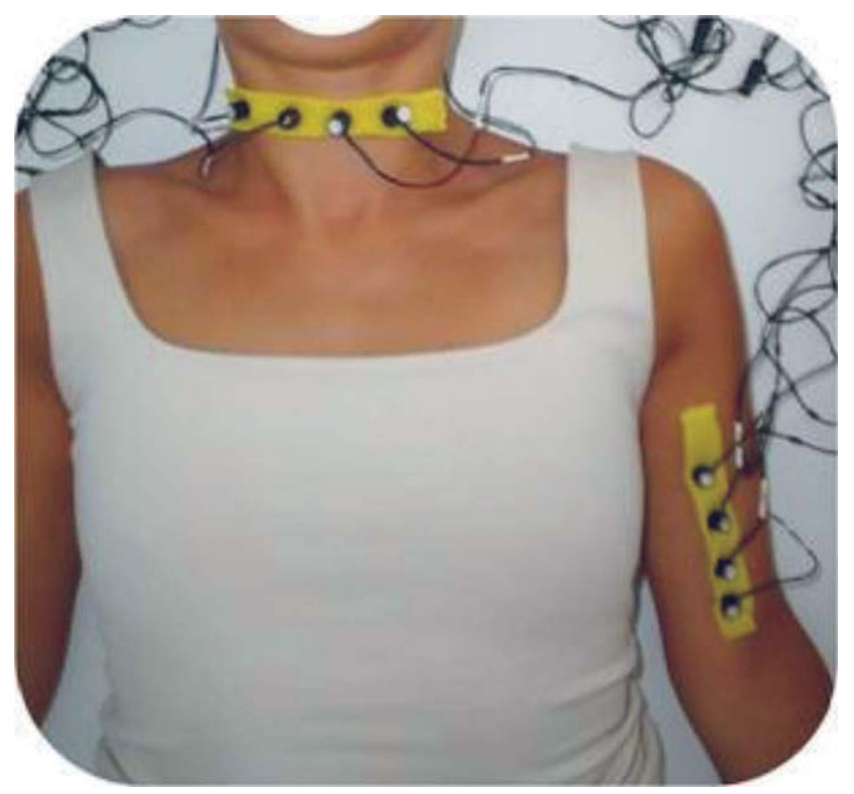

Fig. 2. Solenoid displacement. Subjects judged whether the distance between the active solenoids felt bigger or more intense.

examiner; they were asked to place the pieces one by one to reconstruct the mannequin frontally. No time limit was given. Performance was recorded by a fixed photo camera positioned perpendicular to the test tablet. The camera was controlled by custom made software that captured the images and saved them in a JPEG format $(600 \times 800$ pixel $)$ for further offline coding. The $\mathrm{x}-\mathrm{y}$ pixel coordinates of different critical landmarks (right shoulder, right index finger, right hip and right big toe), which corresponded to the judged locations, were computed using Microsoft Paint 5.1. The coordinates were used to determine the distance between the landmarks. The distance between the shoulder and index finger and between the right hip and the big toe were then converted into centimetres.

\subsection{Peripersonal space task}

In each trial, participants received either a weak (target) or a strong (non-target) electrical stimulus on their right index finger and were instructed to respond vocally (saying "tah"), as quickly as possible, only to the tactile target. Concurrently, a task-irrelevant sound was generated from a loudspeaker nearby (i.e. $5 \mathrm{~cm}$ from the hand, thus within the boundaries of peripersonal space) or a distant loudspeaker. The position of the distant loudspeaker was varied in the two block conditions: in the control condition (far-100), the distant loudspeaker was placed $100 \mathrm{~cm}$ away from the near one, that is, in extrapersonal space; in the critical experimental condition (far-25), the far loudspeaker was placed $25 \mathrm{~cm}$ from the near loudspeaker, that is, just within the putative boundary of the PPS representation (Longo and Lourenco, 2007; Làdavas and Serino, 2008). Although participants were explicitly instructed to ignore the auditory stimulation in this task, we found that nearby sounds, that is, occurring within the PPS, more effectively interacted with tactile stimuli on the hand, fastening tactile reaction time, with respect to distant sounds (Serino et al., 2007, 2011; Bassolino et al., 2010). Thus, we compared the different effects induced by near and far sounds in $\mathrm{MM}$ and controls when the far sounds were presented either at $100 \mathrm{~cm}$ (i.e., within extrapersonal space) or at $25 \mathrm{~cm}$ (i.e. at the PPS boundary). We predicted that in healthy controls, a difference in RTs to near and far sounds would be present only when far sounds were placed at $100 \mathrm{~cm}$ (i.e. well outside PPS) and not at $25 \mathrm{~cm}$ (i.e. within PPS); by contrast, before surgery a near-far difference was evident in MM in both conditions, because sounds presented at $25 \mathrm{~cm}$ fell outside her PPS boundaries. But, considering that the surgical procedure elongated MM's upper limbs by $10 \mathrm{~cm}$, after surgery sounds presented at the same point in space should have been processed as falling within the PPS boundaries if MM had incorporated the elongation 
Table 1

Performance on two point discrimination task (2pdt) and the Von Frey test

\begin{tabular}{lcccc}
\hline & Patient M.M. & Control group $(n$ 26) & Patient M.M. & Control group $(n$ 26) \\
\hline Evaluation & $2 \mathrm{pdt}$ & 2pdt Mean (SD) & Von Frey & Von Frey Mean (SD) \\
PRE & $2.5^{*}$ & $3.54(1.09)$ & $2.44^{*}$ & $2.77(0.27)$ \\
POST & $5.5^{*}$ & $3.46(1)$ & $2.36^{*}$ & $2.75(0.23)$ \\
FOLLOW-UP & $4.5^{*}$ & & $3.61^{*}$ & \\
\hline
\end{tabular}

For both measures smaller value better performances. Note: patient vs. age-matched controls $*<0.001$. Control group were tested only twice.

of her physical body into her mental body representation. If this were the case, no near-far difference would have been found in MM after surgery for sounds presented at $25 \mathrm{~cm}$, as in controls. Tactile stimuli were delivered by two constant-current electrical stimulators (DS7A, Digitimer, Hertfordshire, United Kingdom), via two pairs of neurological electrodes (Neuroline, Ambu, Ballerup, Denmark) placed on the upper side of the index finger. One pair of electrodes delivered the weak stimulus and the other delivered the strong stimulus. Stimuli intensity was titrated for each subject in a pre-test session so that the weak (target) stimulus could be perceived approximately $90 \%$ of the time and the strong (non-target) stimulus could be perceived $100 \%$ of the time. Auditory stimuli were 150-msec bursts of white noise; the intensity of the near and far sounds was set to be equal $(70 \mathrm{~dB})$ as measured by a sound meter at the subjects' ear. The sounds came from two identical loudspeakers: one was located near and one far from the stimulated hand. Inspection of the phono-spectral waves (recorded by a computer) from the two loudspeakers ensured that the sounds were equal at their origin. The tactile and near acoustic stimuli were delivered simultaneously. The far sound had an onset $5 \mathrm{msec}$ before onset of the tactile stimuli to compensate for the delayed arrival of the far sound relative to the near sound, because of its greater distance. RT was measured by means of a voice-activated relay. A computer running XGen (Rorden, n.d.) software was used to control stimulus presentation and record responses. The task was performed in two conditions: far-100 and far25. In each condition, a total of 240 trials, divided into two blocks lasting approximately 6 minutes each, were administered: 60 target trials with the near sound, 60 target trials with the far sound, 40 non-target trials with the near sound, 40 non-target trials with the far sound, and 40 catch trials (i.e. trials in which only a sound, 20 near and 20 far, was presented). To counterbalance the order of condition administration, MM performed the experiment in the following block order: far-100; far-25; far-25; far-100. A group of six age-matched healthy controls (all females, mean age 25 years) performed the same experiment; half performed the task in the same block order as MM, and the other half in the reverse order, far-25; far-100; far-100; far-25.

\section{Results}

\subsection{Primary tactile tasks}

There was no change in the control group's performance on either tactile sensitivity test in the two evaluations (Von Frey, first session $=2.77$, s.e.m. $=0.05 ;$ second session $=2.75$, s.e.m. $=0.05$; $p=0.48 ;$ DPT, first session $=3.54$, s.e.m. $=0.22$; second session $=3.46$, s.e.m. $=0.20 ; p=0.38$ ). On Von Frey's test, MM's performance showed a slight change between prior to surgery (mean diameter 2.44), after surgery (2.36) and at the follow-up (3.61). Nevertheless, the patient's performance was different from that of controls at all three evaluations (all $p<0.001$; see Table 1). MM's 2PD threshold showed a major change across the three testing sessions: before surgery $(2.50 \mathrm{~cm})$, after surgery $(5.50 \mathrm{~cm})$ and at the follow-up $(4.50 \mathrm{~cm})$. In the pretest, MM performed better on the 2PDT when compared with the control group $(t(25)=4.87 p<0.001)$. At the post-test and the follow-up sessions, she performed significantly worse than controls $[t(25)=-10.4 p<0.001 ; t(25)=-5.29$ $p<0.001$ respectively]. In summary, we observed dramatic loss of tactile acuity after surgery.

\subsection{Pressure and distance tasks}

Data were analyzed using point of subjective equality (PSE) and just noticeable difference (JND) for both tasks and compared with one sample $t$-test and paired samples $t$-test using SPSS software analysis (version 16). Data were fitted to a sigmoidal function described by the following equation: 
Table 2

Participants' performance on the Pressure Discrimination task

\begin{tabular}{lcccc}
\hline & Patient M.M. & Control groups $(n$ 20) & Patient M.M. & Control groups $(n$ 20) \\
\hline Evaluation & PSE & PSE Mean (SD) & JND & JND Mean (SD) \\
PRE & $1.54^{*}$ & $0.10(0.34)$ & $-3.70^{*}$ & $-1.81(0.67)$ \\
POST & $1.21^{*}$ & $0.09(0.33)$ & $-0.57^{*}$ & $-1.79(0.66)$ \\
FOLLOW-UP & $0.66^{*}$ & & -1.55 & \\
\hline
\end{tabular}

Note: patient vs. age-matched controls $*<0.001$. Control group were tested only twice.

Table 3

Performance on the Distance Discrimination task

\begin{tabular}{lcccc}
\hline & Patient M.M. & Control groups $(n$ 26) & Patient M.M. & Control groups $(n$ 26) \\
\hline Evaluation & PSE & PSE Mean (SD) & JND & JND Mean (SD) \\
PRE & 0.20 & $0.14(0.26)$ & $-1.97^{*}$ & $-1.53(0.47)$ \\
POST & $1.12^{* *}$ & $0.07(0.31)$ & $-2.16^{* *}$ & $-1.38(0.64)$ \\
FOLLOW-UP & $0.71^{* *}$ & & -1.50 & \\
\hline
\end{tabular}

Note: patient vs. age-matched controls $*<0.01 ; * *<0.001$. Control group were tested only twice.

$$
y=\frac{100}{1+e^{\frac{\left(x-x_{c}\right)}{b}}}
$$

where $\mathrm{x}$ represents the independent variable (i.e., the separation difference between the two points administered on the arm and on the neck) and y the dependent variable (i.e., probability of reporting the distance on the neck as longer). Upper and lower saturation values are fixated at 100 and 0 respectively. $X_{c}$ is the value of the abscissa at the central point of the sigmoid (i.e., the value of $\mathrm{x}$ at which $\mathrm{y}=50$ ) and $\mathrm{b}$ establishes the slope of the sigmoid at the central point. Point of subjective equality (PSE) scores correspond to the $\mathrm{x}_{\mathrm{c}}$ and represent the difference between the two points distance on the arm and the neck yielding to equal probability of perceiving the stimuli separation as longer on the arm or on the neck. Just notable difference (JND) scores are calculated as the difference between $\mathrm{X}_{75}$ and $\mathrm{X}_{25}$, that is between the difference between two points on the arm and the neck yielding to a probability of $75 \%$ and $25 \%$, respectively, of perceiving the two points on the neck as longer. No significant difference was found in the PSE (distance task $p=0.18$, intensity task $p=0.85$ ) and the JND (distance task $p=0.10$, intensity task $p=0.66$ ) in the control group in the two assessments for either the Distance discrimination or the Pressure discrimination task. In the PDt, MM's performance was always significantly different from that of controls at the three different assessments (see Table 2). On the DDt, at the pretest assessment MM's PSE did not differ from that of the control group $[t(25)=1.15, p=0.26]$, whereas her JND was significantly worse $[t(25)=3.47, p<0.001]$.
In the post-test, MM's PSE and JND were significantly different from controls' $[t(25)=17.3, p<0.001$; $t(25)=6.07, p<0.001$ respectively]; this seems to indicate a change in her tactile perception after surgery. At the follow-up assessment, MM continued to show significant differences in PSE but not JND $[t(25)=10.6$, $p<0.001 ; t(25)=0.59, p=0.56]$. When compared with her post-test values, however, her performance tended to be more similar to that of the control group (see Table 3). To summarize, the present results show that after surgery, MM performed steadily on the intensity task but not on the distance task. Specifically, MM perceived the distances on the arm as shorter as they actual were. The peripheral distortion is progressively restored in order to provide a more realistic perception of the size of the stimuli

\subsection{Body image test}

As shown clearly in Fig. 3, before surgery the patient's reconstruction of the body 2D mannequin was different from controls (see also Table 4). However, after surgery and at the follow-up, the shape of the reconstructed 2D mannequin reproduced by the patient was more elongated and similar to that reproduced by the controls. This suggests that the patient perceived the shape of her whole body as elongated after surgical lengthening of the upper limbs alone. To quantify this effect and study it in relationship to the different body parts submitted to surgery, we measured the perceived length of the upper and lower limbs. For this purpose, we calculated the distance between the right shoulder and the right index finger and between the right hip and 
Table 4

Performance on the Body Image test

\begin{tabular}{lcccc}
\hline & Patient M.M. & Control groups $(n$ 16) & Patient M.M. & Control groups $(n$ 16) \\
\hline Evaluation & Right lower limb $(\mathrm{cm})$ & Right lower limb $(\mathrm{cm})$. Mean $(\mathrm{SD})$ & Right upper limb $(\mathrm{cm})$ & Right upper limb $(\mathrm{cm})$. Mean $(\mathrm{SD})$ \\
\hline PRE & 13.99 & $14.41(1.29)$ & $7.43^{* *}$ & $9.85(1)$ \\
POST & 15.36 & $15.15(0.72)$ & $8.73^{*}$ & $9.77(1.12)$ \\
FOLLOW-UP & 15.53 & & 10.3 & \\
\hline
\end{tabular}

Note: patient vs. age-matched controls $*<0.01 ; * *<0.001$. Control group were tested only twice.

Table 5

Performance on the Peripersonal space test

\begin{tabular}{|c|c|c|c|c|c|c|c|c|c|c|c|c|}
\hline & \multicolumn{6}{|c|}{ Patient MM } & \multicolumn{6}{|c|}{ Control group $(n 7)$} \\
\hline & Near 100 & Far 100 & Near-Far & Near 25 & Far 25 & Near-Far & Near 100 & Far 100 & Near-Far & Near 25 & Far 25 & Near-Far \\
\hline$\overline{\mathrm{PRE}}$ & 551 & 579 & -28 & 547 & 563 & -16 & $584(38)$ & $606(40)$ & -22 & $618(35)$ & $612(35)$ & 6 \\
\hline POST & 436 & 452 & -16 & 440 & 433 & 7 & & & & & & \\
\hline
\end{tabular}

Mean RTs (in msec; with s.e.m.) to tactile targets when sounds were presented in near and far space, and the difference between these, in the two experimental conditions.

the right big toe from the figures reconstructed by the patient and the controls.

As can be seen in Table 4, before surgery MM perceived her upper limbs as shorter $(7.43 \mathrm{~cm}) \mathrm{com}$ pared with her aged-matched controls $(9.85 \mathrm{~cm})$ $[t(15)=9.59, p<0.001]$; even after surgery the performance of MM remained significantly different from those of controls $[8.73 \mathrm{~cm}$ patient; $9.77 \mathrm{~cm}$ control group; $t(15)=3.67, p=0.002$ ] and it was no more

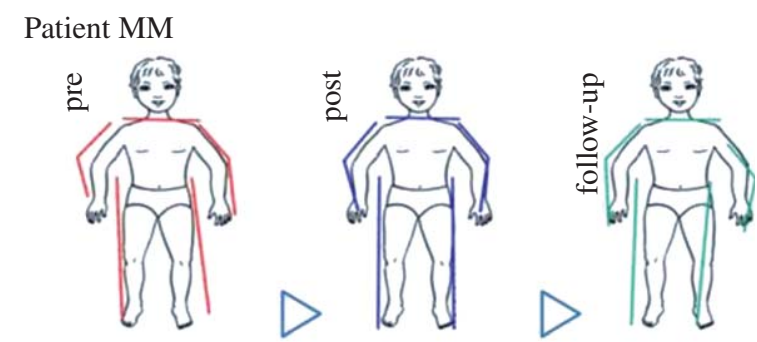

Control group (n 16)
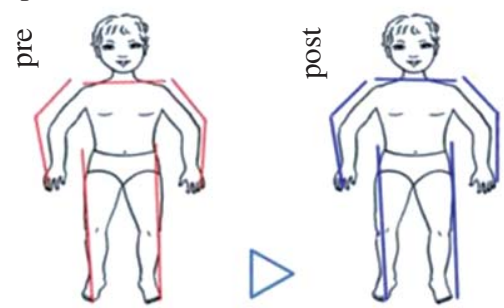

Fig. 3. MM's performance on the DH test. The position of the arms and legs of the mannequin is represented graphically by the lines. After surgery (post/follow-up), MM positioning her arms similarly to controls. No difference was found in the positioning of the legs between the three assessments. Control group were tested only twice. significant at the follow-up $[10.3 \mathrm{~cm} ; t(15)=-1.88$, $p=0.79]$. By contrast, no significant change was found in the three assessments for perceived length of the lower limb, which was consistently different from that of controls [MM pre $13.99 \mathrm{~cm}$, control group $14.41 \mathrm{~cm}$, $t(15)=1.33, p=0.20 ; \mathrm{MM}$ post $15.36 \mathrm{~cm}$, control group $15,15, t(15)=-1.17, p=0.26$; MM follow-up $15.53 \mathrm{~cm}, t(15)=-2.1, p=0.053]$. No change in the perceived dimensions of the upper and lower limbs was found in the healthy controls at the two assessments (upper limbs $p=0.82$; lower limbs $p=0.06$ ). Thus, results from the DH task suggest that physical elongation of the upper limbs was incorporated into the mental body representation, so that it selectively shaped the perceived length of the upper limb.

\subsection{PPS task}

MM's accuracy was extremely high. Omissions and false alarms were very low (on average 1.62 and 0.87 per block, respectively), and thus were not analyzed. Mean RTs to tactile stimuli were computed and compared for the different experimental conditions (see Table 5).

RTs above two standard deviations from the mean were trimmed from the analysis ( 0.75 trials per block, on average). Before surgery, MM responded faster to a tactile stimulus on the hand when a sound was presented near rather than far from the hand, both when the far sound was presented at $100 \mathrm{~cm}$ (far-100 condition: near $=551$; far $=579)$ and at $25 \mathrm{~cm}$ (far-25 condition: RTs associated with near sounds $=547 \mathrm{~ms}$; 
RTs associated with far sounds $=563 \mathrm{~ms}$ ), with a nearfar RT difference of -28 and $-16 \mathrm{msec}$, respectively. In healthy controls, faster RTs associated with near sounds compared with far sounds were evident only in the far-100 condition, with a near-far difference of -22 , and not in the Far- 25 condition, with a nearfar difference of $7 \mathrm{msec}$. A $2 \times 2$ ANOVA with the factors sound position (near and far) and condition (far100; far-25) revealed a significant two-way interaction sound position $\mathrm{X}$ condition $[\mathrm{F}(1,6)=20.45 ; p<0.01]$, confirming that the speeding effect due to near sounds was present in the Far-100 condition (near $=584 \pm 38$; far $=606 \pm 40 ; p<0.01$ ) but not in the far- 25 condition $($ near $=618 \pm 35 ;$ far $=612 \pm 35 ; p=0.33)$. Thus, in the far-100 condition a similar space-dependent modulation of RTs was present in MM before surgery and in controls $(\mathrm{MM}=-28$; controls $=-22$; one sample $t$-test on near-far RT difference: $t(6)=1.90 ; p=0.10)$. Instead, in the far-25 condition, a near-far RT difference was evident in MM but not in controls $(\mathrm{MM}=-16$; controls $=6$; one sample $t$-test on nearfar RT difference: $t(6)=3.95 ; p<0.01$ ). After surgery, MM still showed faster RTs to tactile stimuli paired with near sounds (436) compared with those paired with far sounds (452) in the far-100 condition, with a near-far RT difference $(-16 \mathrm{msec})$ comparable to that found in healthy controls $[t(7)=1.79, p=0.12]$. However, differently from before surgery, in the far- 25 condition, RTs associated with near sounds (440) were no faster than those paired with far sounds (433), with a near-far difference of $7.5 \mathrm{msec}$, which was not different from that of healthy controls $[t(7)=-29, p=0.78]$. To sum up, both in MM (before and after surgery) and in controls, a sound presented $5 \mathrm{~cm}$ away from the hand had a stronger effect on the processing of a tactile stimulus on the hand than a sound presented at $100 \mathrm{~cm}$ away, suggesting a stronger multisensory interaction effect when both the tactile and auditory stimulus occurred within PPS. No near-far difference was present in healthy controls when far sounds were administered $25 \mathrm{~cm}$ from the near stimulus, that is, when both sounds were presented within the putative PPS boundary. This was not true for MM before surgery, when a sound near her hand fastened tactile RTs compared with a sound presented $25 \mathrm{~cm}$ away, suggesting that a sound at that distance did not interact with tactile processing of the hand. However, after the surgical procedure had lengthened MM's arm by $10 \mathrm{~cm}$, the difference between the effect due to near and far sounds vanished when the far sound was presented
$25 \mathrm{~cm}$ away and, analogously to controls, was still present when the far sound was presented $100 \mathrm{~cm}$ away. This finding suggests that after surgery the same spatial position was processed as closer to the hand than before surgery, as if the space where the touch on the hand and the sound interacted was extended after the arm was physically lengthened.

\section{Discussion}

Surgical extension of the upper limbs of an achondroplasic young woman resulted in changes in several levels of body representation. This finding supports the view that body representations are dynamic constructs remodelled by experience throughout life. In particular, the brain's maps of the body surface, and more cognitive representations of body form must both adapt to normal changes in the physical body throughout the lifespan, notably in childhood growth and in ageing. However, these processes have proved difficult to investigate experimentally. Changes in the physical body are normally so small or so slow that the effects are difficult to quantify. Perhaps as a result, the scientific literature on neural representation of the body has relied on transient illusory effects, particularly experimentally-induced changes in the perceived size of body parts (see e.g. Longo et al., 2010 for a review). Such illusions are valuable in showing what sources of information contribute to the representation of the body, but cannot reveal how gradual changes in body configuration lead to changes in body representation. Here, for the first time, we investigated plasticity in body representations after an actual and permanent change in the structure of the physical body and in the absence of any disconnections between the periphery and the cortex. Overall, the results indicate that the primary level of somatosensory processing are less affected by elongation surgery than higher levels of body representations, which underwent selective alterations soon after the elongation, and progressively re-adapted at 11 months post surgery.

\subsection{Primary tactile tasks}

In the first series of experiments, we investigated whether body part elongation leads to modifications at different levels of stimulus processing, that is, from primary somatosensation to higher levels of body representation. In this hierarchical view, we found that 
arm elongation did not affect the most primary sensory process of detecting tactile stimulation, since it left unaltered the patient's ability to detect the presence of a tactile stimulus on the arm, as measured by the Von Frey test. However, tactile spatial acuity (as assessed by the 2PDt) decreased after elongation and became worse than that of healthy controls. This finding could simply reflect peripheral effect of stretching the skin. Since tactile acuity strongly follows the density of skin innervations by mechanoreceptive afferents, decrease in tactile innervations density caused by skin stretch should produce a decrease in tactile acuity. However it is impossible to determine from our data, whether stretching the skin requires some additional central adaptation of somatosensory processing. It is important to highlight that previous research documented that after Ilizarov surgery a partial denervation in the motor distribution of the deep nerves can occurs and that this consequence does not affects sensory conduction (Galardi et al. 1990). Moreover patient MM didn't suffered from any kind of denervation so we can strongly support the issue regarding the integrity of the basic afferent pathways. Direct assessment of skin innervation before and after surgery by skin biopsies would be required to distinguish central from peripheral explanations, but this was not ethically appropriate in this case.

\subsection{Distance and pressure discrimination tasks}

At a subsequent step of processing, we observed that the elongation surgery affected the patient's ability to process two contacts on the surface of the arm when she had to focus on the metric properties of the stimuli on the skin, but left unaffected her ability to judge the pressure intensity of the same contacts on the same body location. One year post-surgery, her sensitivity to pressure remains stable, whereas her metric perceptual functions have improved toward her pre-surgery evaluations. The dissociation between these two forms of touch perception was investigated in a previous fMRI study (Spitoni et al. 2010), where it was found that the same tactile stimuli requiring either spatial distance judgement or contact pressure judgement bilaterally activated parietal and frontal areas. However, spatial distance evaluation on the body surface also selectively activated the angular gyrus and the temporo-parietooccipital junction in the right hemisphere. They interpreted these results as the need to refer tactile stimulations to a metric body representation in the tactile distance judgement task, whereas judging contact pressure can be performed without this representation. In the case of MM, it seems that surgical modification of the arms selectively altered tactile judgements only when these required mediation by body representation. Compared to control group, she perceives, after stretching, tactile stimuli on the arm as closer, while in the follow-up she tends to return towards the pre surgery discrimination. This result can be related to Weber's studies (1996) on tactile illusions. The author found that the perception of tactile distances were related to tactile sensitivity and that the size distortion derives from density of the mechanoreceptors on the skin. We can speculate that the sudden elongation of MM's arms led to a kind of diffusion of the mechanoreceptors toward the entire area of the stretched skin; in other words the same amount of receptors that the system used before the elongation, were now utilized to cover a larger area. This effect could partially account for why MM perceived stimuli as closer. Conversely, at follow-up evaluation we observed that the distance judgments were more veridical than post surgery. This suggests that additional process of tactile size constancy are required to correct the distortions inherent in primary representations. The need for such additional processes has been also suggested by Longo et al. (2010), who describe two classes of higher-order processing beyond the preliminary somatosensation: somatoperception and somatorepresentation. Obviously in this case we refer to the former which dealsto the process of perceiving the body and ensuring the constancy of somatic percept.. Summarizing, after intervention, we observe that the perception of distance between two points decreases, but subsequently the metric properties of the stimuli are scaled by somatoperception which correcting such a distortions.

\subsection{Body image test}

On the other hand, we can also describe MM's performance on the DH Body Image Test as an adjustment of somatorepresentation. Before the elongation, MM's body representation (as measured by the $\mathrm{DH}$ test) was consistent with the shape of her own body at the time. Specifically, MM exhibited a selective bias in reconstructing the shapes and dimensions of the upper, whereas the lower limbs were within the normal range. After surgery, her performance improved, and at the follow-up her reproduction of the shape of the body was similar to that of the healthy controls. 
This normalization effect could be seen in MM's placement of the tiles reproducing the upper limbs as if they were longer. No such change occurred in her placement of the lower limb tiles. The patient had previously undergone elongation surgery for her lower limbs, and this may be the reason why her placement of the leg tiles was similar to that of the controls. This pattern of results suggests that the elongation of the arms was specifically incorporated in an updated representation of the body, without changes in representations of the rest of the body. This evidence supports the finding of a previous study (Di Russo et al., 2006) in which we observed that the body representation of achondroplasics was partially restored after elongation. Given this evidence, we can speculate that achondroplasics' preexisting body representation can be modified towards a body template more similar to that of healthy controls when surgical reconstruction is used to change limb size.

\subsection{Peripersonal task}

Finally, elongation surgery also affected the patient's PPS representation. We used the differential effect of near and far auditory stimuli on tactile processing (Serino et al., 2007; Bassolino et al., 2010) as a probe of the extension of PPS around the arm. For MM before surgery and for healthy controls, a sound administered close to the hand resulted in faster tactile RT compared to a sound presented $100 \mathrm{~cm}$ away, in extrapersonal space. This near-far difference was taken as evidence of stronger audio-tactile interaction for stimuli falling within PPS. In keeping with this, in healthy controls a far sound presented just inside the PPS boundary (i.e. at a distance of about $25 \mathrm{~cm}$ ) affects tactile RT similarly to a near sound. This was not the case when MM was tested before surgery: then near sounds induced faster RTs than far sounds administered at $25 \mathrm{~cm}$, suggesting that stimuli presented at $25 \mathrm{~cm}$ fell outside her PPS boundary. Importantly, after MM's arm was lengthened by about $10 \mathrm{~cm}$, the near-far difference was abolished for sounds presented at $25 \mathrm{~cm}$ but not for sounds presented at $100 \mathrm{~cm}$, so that MM's behaviour was analogous to that of healthy controls. This finding suggests that once MM's arm was elongated, the PPS boundary shifted to include a portion of space that is part of the PPS of healthy individuals. Longo and Lourenco (2007) used a line bisection task, in which lines were presented at different distances from the subject, to measure the extension of PPS in healthy adults.
They found a correlation between the physical length of the arm and the extension of PPS as measured by the line bisection task, suggesting that arm length constitutes a metric for representing the space around the body. Our results confirm this finding and also show that the relationship between body part dimensions and PPS representation is dynamic and updates to changes in the physical body. This mechanism might be particularly relevant during development, when the brain needs to keep track of the continuous changes in body dimensions due to growth and also to plan and correctly execute actions toward objects placed at different distances from the body.

\section{Conclusions}

Some final considerations are needed to qualify the plasticity of somatoperception and somatorepresentation. The forms of plasticity demonstrated in the present study are selective in several ways. First plasticity is selective for specific perceptual processing: pressure evaluation on the skin is not affected by surgical elongation, but distance perception is significantly modified. Second, plasticity is confined to the modified body segment. This part-specificity rules out accounts based on general factors such as perceptual learning, or non-specific effects of surgery. Although this observation would be trivial in the case of the illusory elongation produced by tendon vibration (de Vignemont, 2005), it is not so in our case. A $10 \mathrm{~cm}$ increase in the length of the arms produces considerable improvement in the ability to explore external space (i.e. grasping, throwing, pointing etc.). The changes were also space-specific: the effect of the audio-tactile interaction indicates a change in peripersonal but not extrapersonal space. Similarly, the capacity to represent different body segments to form a complete body image (DH test) showed significantly improvement regarding representation of the relationship between the arms and the body, but left unchanged the relationship between the leg and the body. These plastic effects involving high-order body representations require time, and some plasticity phenomena occurred months after the elongation procedure ended.

To summarise, we have documented changes in several levels of bodily awareness as a result of a surgical elongation procedure in a single case.

We found improvements in measures of primary tactile sensation (detection, intensity coding), which 
were not necessarily sustained at follow-up. Changes in tactile spatial acuity were consistent with a plastic change in receptive fields. Immediately after surgery, tactile acuity was decreased, presumably reflecting a decrease in mechanoreceptor density due to the extended skin area. This was reversed at follow up, presumably reflecting a reorganisation of receptive field territories. Finally, we found major changes at the levels of somatoperception and somatorepresentation. An explicit body image task showed rapid and sustained adjustment to the elongation. We also found an immediate expansion of the zone of peripersonal space, as measured by multisensory interactions.

Overall, our results suggest that most aspects of somatosensory awareness show considerable plasticity when the body itself changes. Such plasticity is generally assumed in development. MM's performance suggests that the same plasticity persists in adulthood. In this sense, this case offers a unique window into how the brain tracks the state of the body, and adjusts perceptual mechanisms accordingly.

\section{Acknowledgments}

PH was supported by EU FP7 project VERE, and by a Leverhulme Trust research fellowship. AS was funded by Volkswagen Stiftung, the (Un)Bound Body Projects.

\section{References}

Basso, A., Gardelli, M., Grassi, M.P., \& Mariotti, M. (1989). The role of the right hemisphere in recovery from aphasia. Two case studies. Cortex, 25(4), 555-566.

Bassolino, M., Serino, A., Ubaldi, S., \& Làdavas, E. (2010). Everyday use of the computer mouse extends peripersonal space representation. Neuropsychologia, 48(3), 803-811.

Cattaneo, R., Villa, A., Catagni, M., \& Tentori, L. (1988). Limb lengthening in achondroplasia by Ilizarov's method. Inter Orthop, 12, 173-179.

Daurat-Hmeljiak, C., Stambak, M., \& Berges, J. (1978). Il test dello schema corporeo. Una prova di conoscenza e costruzione dell'immagine del corpo. Hove: Org Spec Firenze.

de Vignemont, F., Ehrsson, H.H., \& Haggard, P. (2005). Bodily illusions modulate tactile perception. Curr Biol, 26, 1286-1290.

Di Russo, F., Committeri, G., Pitzalis, S., Spitoni, G., Piccardi, Pizzamiglio, L., et al. (2006). Cortical plasticity following surgical extension of lower limbs. Neuroimage, 30(1), 172-183.

Galardi, G., Comi, G., Lozza, L., Marchettini, P., Novarina, M., Paronzini, A., et al. (1990). Peripheral nerve damage during limb lengthening. Neurophysiology in five cases of bilateral tibial lengthening. J Bone Joint Surg Br, 72(1), 121-124.

Gerstmann, J. (1940). Syndrome of finger agnosia, disorientation for right and left, agraphia and acalculia. Arch Neurol Psychiatr, 44, 398-406.

Gerstmann, J. (1957). Some notes on the Gerstmann syndrome. Neurology, 7(12), 866-869.

Graziano, M.S., \& Cooke, D.F. (2006). Parieto-frontal interactions, personal space, and defensive behaviour. Neuropsychologia, 44(13), 2621-2635.

Ilizarov, G.A., \& Deviatov, A.A. (1971). Surgical elongation of the leg. Ortop Travmatol Protez, 32, 20-25.

Lackner, J.R. (1988). Some proprioceptive influences on the perceptual representation of body shape and orientation. Brain, $111(\mathrm{Pt}$ 2), 281-297.

Làdavas, E. \& Serino, A. (2008). Action-dependent plasticity in peripersonal space representations. Cogn Neuropsychol, 25(78), 1099-1113.

Levine, D.N., \& Mohr, J.P. (1979). Language after bilateral cerebral infarctions: Role of the minor hemisphere in speech. Neurology, 29(7), 927-938.

Longo, M.R., \& Lourenco, S.F. (2007). Space perception and body morphology: Extent of near space scales with arm length. Exp Brain Res, 177(2), 285-290.

Longo, M.R., Azañón, E., \& Haggard, P. (2010). More than skin deep: Body representation beyond primary somatosensory cortex. Neuropsychologia, 48(3), 655-668. Review.

Oldfield, R.C. (1971). The assessment and analysis of handedness: The Edinburgh inventory. Neuropsychologia, 9(1), 97-113.

Rizzolatti, G., Fadiga, L., Fogassi, L., \& Gallese, V. (1997). The space around us. Science, 277(5323), 190-191.

Schwoebel, J., \& Coslett, H.B. (2005). Evidence for multiple, distinct representations of the human body. JCogn Neurosci, 17(4), 543-553.

Semenza, C. (1988). Impairement of localization of body parts following brain damage. Cortex, 24, 443-449.

Serino, A., Bassolino, M., Farnè, A., \& Làdavas, E. (2007). Extended multisensory space in blind cane users. Psych Sci, 18(7), 642648.

Serino, A., Canzoneri, E., \& Avenanti, A. (2011). Fronto-parietal areas necessary for a multisensory representation of peripersonal space in humans: An rTMS study. J Cogn Neurosci, 23(10), 2956-2967.

Sirigu, A., Grafman, J., Bressler, K., \& Sunderland, T. (1991). Multiple representations contribute to body knowledge processing. Evidence from a case of autotopagnosia. Brain, 114(Pt 1B), 629-642.

Spitoni, G.F., Galati, G., Antonucci, G., Haggard, P., \& Pizzamiglio, L. (2010). Two forms of touch perception in the human brain. Exp Brain Res, 207(3-4), 185-195.

Taylor-Clarke, M., Jacobsen, P., \& Haggard, P. (2004). Keeping the world a constant size: Object constancy in human touch. Nat Neurosci, 7(3), 219-220.

Weber, E.H. (1996). De subtilitate tactus. In H.E. Ross. \& D.J. Murray (Eds.), E.H. Weber on the tactile senses. Hove, East Sussex, Erlbaum, (original work published 1834). 